\title{
Diagnosis and treatment of participants of support groups for hypersexual disorder
}

\author{
Els Tierens, Johan Vansintejan, Jan Vandevoorde, Dirk Devroey \\ Department of Family Medicine, Vrije Universiteit Brussel, Belgium.
}

\begin{abstract}
Summary Background: The aim of this study is to examine the extent to which members of support groups for hypersexual disorder meet the proposed criteria for hypersexual disorder of Kafka, how the diagnosis of hypersexual disorders is made and what treatments are currently given.

Methods: In this non-interventional research survey, members of support groups for hypersexual disorder received a questionnaire in which the criteria for hypersexual disorder according to Kafka were included as well as the way the disease was diagnosed and treated.

Results: The questionnaire was presented to 32 people but only 10 completed questionnaires were returned. Five of the ten respondents met the criteria of Kafka. For the other five respondents a hypersexual disorder was not confirmed but neither excluded. Only for three respondents the diagnosis was made by a professional healthcare worker. The treatment included - besides the support group in nine cases - also individual psychotherapy. Two respondents took a selective serotonin re-uptake inhibitor (SSRI), as recommended in the literature.

Conclusions: The members of support groups for sex addiction were difficult to motivate for their participation. The way hypersexual disorders were diagnosed was far from optimal. Only two participants received the recommended medication.
\end{abstract}

KEY WORDS: Sexual disorders, addiction, treatment.

Submitted 4 November 2013; Accepted 15 January 2014

\section{INTRODUCTION}

\section{Definition}

Hypersexual disorder, better known as "sex addiction" is a clinical phenomenon that has received only little attention from researchers up to now. Hypersexual disorder was first introduced in the Diagnostic and Statistical Manual of Mental Disorders (DSM)-III. The DSM-III-R specified that hypersexual disorder was different from paraphilia. In the DSM-IV hypersexual disorder was removed because there was a great lack of empirical research and consensus on definition, aetiology and pathogenesis (1). In the DSM-IV hypersexual disorder was placed under "Sexual Disorder Not Otherwise Specified". It was specified as "distress about a pattern of repeated sexual relationships involving a succession of lovers who are experienced by the individual only as things to be used" (2).

The proposal to include the condition as "sex addiction" to the DSM-V was rejected for similar reasons. There is still disagreement whether such a condition really exist as a separate entity. It may be a manifestation of another psychiatric disorder (3).

Kafka suggested in 2010 to include hypersexual disorder as a new psychiatric disorder in the DSM-V (1). The name does not impose any causal link or does not suggest any particular pathogenesis. The criteria (Figure 1)

\section{Figure 1.}

Proposed criteria

for hypersexual disorder (2) used in our study.

A. Over a period of at least 6 months, recurrent and intense sexual fantasies, sexual urges, or sexual behaviors in association with 3 or more of the following 5 criteria:

A1. Time consumed by sexual fantasies, urges or behaviors repetitively interferes with other important (non-sexual) goals, activities and obligations.

A2. Repetitively engaging in sexual fantasies, urges or behaviors in response to dysphoric mood states (e.g., anxiety, depression, boredom, irritability).

A3. Repetitively engaging in sexual fantasies, urges or behaviors in response to stressful life events.

A4. Repetitive but unsuccessful efforts to control or significantly reduce these sexual fantasies, urges or behaviors.

A5. Repetitively engaging in sexual behaviors while disregarding the risk for physical or emotional harm to self or others.

B. There is clinically significant personal distress or impairment in social, occupational or other important areas of functioning associated with the frequency and intensity of these sexual fantasies, urges or behaviors.

C. These sexual fantasies, urges or behaviors are not due to the direct physiological effect of an exogenous substance (e.g., a drug of abuse or a medication).

Specify if:

- Masturbation

- Pornography

- Sexual behavior with consenting adults

- Cybersex

- Telephone sex

- Strip clubs

- Other: 
were drawn up based on a thorough literature review: there must be a disorder of sexual desire and a problem of loss of control over impulses that leads to negative consequences. None of the proposed theories such as disinhibiting of behavior, impulsivity, compulsivity or addiction were proven, but they overlapped. These criteria are not yet tested on a population (1).

Currently, the American Psychiatric Association investigates whether or not hypersexual disorder should be included in DSM-V (4).

Several studies among people with hypersexual disorder showed that 35 to $73 \%$ practiced excessive masturbation, 49 to $51 \%$ frequently searched pornography, in 13 to $70 \%$ consenting adults are involved and $24 \%$ practiced phone sex (1, 5-7).

Kafka also added strip clubs and cybersex to the specifications. However, this cannot be supported by the literature (1).

\section{Differential diagnosis}

Hypersexuality can also fit in many other psychiatric disorders (3). The most common disorders in the differential diagnosis are paraphilias, sexual disorders not otherwise specified, impulse control disorder not otherwise specified, bipolar affective disorder (type I or II), posttraumatic stress disorder and adjustment disorder (disturbance of conduct).

Rather infrequent disorders are substance-induced anxiety disorder (obsessive-compulsive symptoms), substance-induced mood disorder (manic features), delusional disorder (erotomania), obsessive-compulsive disorder, gender identify disorder and finally delirium, dementia, or other cognitive disorders.

\section{Comorbidity}

Hypersexual disorder can result in many other problems such as sexually transmitted diseases, unwanted pregnancy, relationship- and marital problems and domestic violence. It may also have legal consequences (8).

Hypersexual disorder is associated with other psychiatric disorders and risky behaviors such as smoking, excessive drinking, illegal drug use and gambling $(5,9)$.

Professionals specialised in addiction often look for sexually compulsive behavior after identifying a substance dependence. Sex addiction is often associated with substance dependence and is a frequent cause of relapse. This would occur in 39 to $45 \%$ of sex addicts. Mainly cocaine, alcohol and metamphetamines are concerned. Only 17 to $34 \%$ of the surveyed population had no other addiction. Different addictions can occur simultaneously, reinforce and alternate each other as well $(3,10)$.

Within a homosexual population, $45 \%$ of those who scored high on sexual compulsivity frequently used alcohol during sex, in contrast to the non-compulsive group in which this was 39\%. For drugs this was $37 \%$ and $28 \%$ respectively (11).

\section{Epidemiology}

There are no reliable epidemiological data on hypersexual disorder available. The lack of consensus on a definition and on an empirically validated instrument hampers further research and collection of epidemiological data.
For that reason, no large-scale studies have taken place. $(3,5)$ It is estimated that 3 to $6 \%$ of the general population has a hypersexual disorder. It seems to be more common in men than in women; the male-female ratio is estimated at three to five (8).

There is an issue of over-and underestimation. An overestimation could be caused by the current social climate and the perception of sexuality. The popularisation of the concept of hypersexual disorder could also play a role. An underestimation is due to shame, secrecy and depression that people refrain to seek professional help (8). The number of sex addicts that looked for help in Flanders has increased exponentially in the recent years.

In $2010,14,396$ people participated in a study on human sexuality. Of the 6,458 men in the study, 107 (1.7\%) ever searched help for sexual compulsivity. Of the 7,938 included women 69 (0.9\%) searched help. The help-seeking men watch more pornography than women. The women seeking help had more psychological symptoms such as depression and anxiety. The study population is not representative because the participants were recruited in the United States and Canada through internet sites that provide sexual advice (12).

In New Zealand, 940 people at the age of 32 were questioned on excessive sexual behavior and risky sexual behavior. In total 3.8\% men and $1.7 \%$ women reported excessive sexual behavior that interfered with their lives in the past year. They rarely sought help for their sexual behavior. If so, they consulted a psychiatrist, a psychologist, a lawyer or a priest (13).

In a Swedish study with 2,450 people aged between 18 and 60 years, 12\% men and 7\% women had "high hypersexuality". This study enquired about masturbation, use of pornography, number of sexual partners, adultery, multiple sexual relationships at the same time and group sex (9). In 1993, a study recruited people with compulsive sexual behavior through newspapers. In total 36 participants (28 men and 8 women) with sexual preoccupation or excessive sexual behavior and subjective suffering were included. Their medical history showed in 39\% of the cases depression, phobia in $42 \%$ and in $64 \%$ substance addiction. The average age of onset of the hypersexual disorder was 18 years and participants suffered on average since nine years. Three quarters linked this behaviour to the use of alcohol or drugs (14).

\section{Treatment}

Persons with hypersexual disorder more often visit a doctor for sexual advice (9). Carnes already emphasised the importance of the first line in the detection and counselling of people with an addictive sexual dysfunction (10).

If the sexual compulsive behavior is secondary to an addiction or another psychiatric condition, then the latter should be treated first (3). In addition to education, a combination of individual psychotherapy and group therapy is indicated.

An early start with the 12-step program based on Alcoholics Anonymous is highly recommended. Several support groups, where peers meet, follow this pattern. These sessions take place without a therapist $(3,5,10)$. The effect of the 12-step program has not yet been demonstrated but $23 \%$ would complete the first nine 
steps in 18 months and among them relapse is rare. In the beginning, for 30 to 90 days, total abstinence is recommended. This period may be associated with acute depression, insomnia, irritability, difficulty concentrating, and nausea. The symptoms would only emerge in the first 3 weeks and improve during the two months thereafter. Later participants comply with this total abstinence or turn it over to a partial abstinence. This means a total denial of compulsive, destructive sexual behavior. The abstinence means not only a change of behavior but also the avoidance of fantasies (3).

In a second phase individual psychotherapy is initiated: cognitive-behavioral and psychodynamic therapy are recommended $(3,5,10)$. The effect of this therapy is not yet proven, because it is very difficult to organise a randomised controlled trial because of the complexity of the interaction between the caregiver and the patient (15). The intervention of sexologists would be most effective at a later stage, in the second year and later (3). Also couple or family therapy can be added. There are no empirical studies that demonstrate the effect of couple therapy, but partners of addicts are demanding more support from therapists (16).

Reasons to choose for an inpatient treatment are: suicidal tendencies, little social support, failure of outpatient treatment, multiple addictions and serious consequences (legal, financial, marriage-bound or public exposure). Outpatient treatment may be successful when patients are supported by their family. One of the best predictors of success is the will of the patient to succeed (10).

Although the etiology is unknown, researchers focus on the neurophysiology of sexual arousal that depends on neurological, hormonal and genetic factors. But in humans, culture and context play also a major role $(9,10)$.

Patients with pronounced symptoms are advised to start with psychotherapy and medication at the same time because a combination of both gives better results. A therapy with SSRIs is preferred although tricyclic antidepressants are also prescribed (15).

For the more severe cases, particularly offenders, a combination of an SSRI with an anti-androgen (cyproterone acetate or medroxyprogesterone acetate) is preferred but LHRH agonists and estrogens are also used (17).

Evidence about the drug treatment is not available. The study populations are often too small or non-representative population are examined $(3,15,17)$.

Benzodiazepines are not recommended, as these can disinhibit patients (5).

For these kind of disorders there is always a risk of relapse. In the first year there is a great agitation but the following six months include the greatest risk. Only after 18 months an improvement in quality of life occurs and in the fourth and fifth year relationships can improve. A final recovery is, in principle, never reached (3).

\section{Aim of the study}

The aim of this study is to examine the extent to which members of support groups for sex addiction meet the proposed criteria for hypersexual disorder of Kafka. Secondly, the authors try also to have a better insight on how the diagnosis of hypersexual disorders is made and what treatments are currently provided.

\section{Methods}

\section{Participating support groups}

Sexaholics anonymous (SA), sexual compulsives anonymous (SCA) and sex and love addicts anonymous (SLAA), the three support groups active in Belgium were invited to participate in the study. Only SA and SCA decided to participate. The SLAA preferred not to contribute to the research because during the meeting they just want to concentrate on the treatment.

SA was founded in 1979 in the United States. In 2011, SA had 1611 groups in 42 countries. In Belgium there are currently four SA groups. In the course of the four years in which they have operated, there are a few thousand people who attended at least once and about one hundred who attended regularly the meetings. Most of the people (95\%) attend only once. In December 2011, there were five regular members in each of the four groups.

The meetings are chaired by the members themselves, in no SA-meeting professional counsellors are present. SCA reported that in their support groups 15 to 20 members worked on their recovery. There is no register of members, so they cannot provide exact figures.

\section{Procedure}

Non-interventional research was organised in March 2012. The questionnaire was anonymously proposed to members of the participating support groups for sex addiction. The questionnaire consisted of a paper and electronic form and was distributed by the contact person of each support group. The questionnaire was available in Dutch as well as in French.

After the collection and the analyses of data, the contact persons of the support groups were interviewed in order to clarify and comment the results.

\section{Ethical approval}

The protocol for this study was approved by the Ethical Committee of the University Hospital of the Vrije Universiteit Brussel. The participants were informed about the purpose and course of the study, possible risks, confidentiality and the right to information, on the front page of the questionnaire.

Informed consent was obtained in an alternative manner. By participating in the study and by completing the questionnaire the subject confirmed that he/she was aware of the purpose and course of the study and possible risks. This was the only way to guarantee absolute anonymity.

\section{Questionnaire}

The questionnaire consisted of three parts. In a first part, some socio-demographic data were collected. The criteria for hypersexual disorder were collected in the second part. The last section included questions related to diagnosis, treatment and satisfaction with treatment. The socio-demographic variables in the questionnaire were: sex, age, highest degree, ethnicity, sexual orientation and relationship status.

To assess whether the members of the support groups actually meet the criteria of Kafka a newly developed ques- 
tionnaire that reflects these criteria as strong as possible was administrated. There were already several questionnaires used for disease screening and diagnosis. However, these questionnaires were not studied in detail and none of them specifically sets the criteria of $\operatorname{Kafka}(18,19)$. They were therefore not eligible for our study.

In the third part, participants were asked which health professional made the diagnosis, which professionals did the treatment follow-up, what was the treatment and what the medication was the patient received. It was also checked whether a physician - if any - was involved. Finally, the participants were asked for how long they already suffered from hypersexual disorder, for how long the disease was already diagnosed and whether they were satisfied with the treatment so far.

\section{Statistical analyses}

All used statistics are descriptive. IBM SPSS 20 was used for the analyses.

\section{Results}

\section{Demographics}

The contact person of SA presented the questionnaire to 25 members and 7 completed questionnaires were returned. The contact person of SCA presented the questionnaire to 7 members and 3 completed questionnaires were returned. The response rate was 31\%.

All participants were male. Their ages ranged between 33 and 62 years and averaged 48.2 years. They were all Caucasians except two. One participant was partly South American and partly Caucasian. The other participant did not answer this question.

Most of the participants obtained a degree of non-university higher education. All participants of the study were heterosexual except one who was homosexual. Seven participants had a partner, the other three had no relationship.

\section{Diagnosis}

Five out of 10 participants met the three criteria of Kafka. All respondents met the first criterion and nine met also the second criterion (Table 1). Five participants were addicted to substances.

The sexual activity of all respondents took place on the field of masturbation and pornography. Six respondents had regular sex with consenting adults, four respondents took part in cybersex, one in phone sex and three participants regularly visited strip clubs. Four respondents reported other inappropriate behavior like voyeurism in public places, cinemas, nudist beaches and swingers clubs.

The diagnosis was made by a professional healthcare worker in three cases, which was in each of these cases a psychologist. One of these three psychologists was an expert in hypersexual disorder by experience. Three respondents made the diagnosis themselves, twice the diagnosis was made by the support group and twice the partner made the diagnosis.

On average the respondents suffered for 31 years of hypersexual disorder. The age at which it started varied between 4 and 24 years, with an average of 17.5 years, but the diagnosis was on average made at the age of 37 years.

\section{Treatment}

Eight participants had a family physician. All family physicians (except one) were aware of the diagnosis. Four of these eight family physician were involved in the treatment. Six participants were treated by a psychologist, four by a sexologist and five by a psychiatrist. One participant indicated that "the literature" was responsible for his treatment. No one sought help from a support group only.

The treatment included ambulatory individual psychotherapy in nine participants, two followed relational therapy, one followed an online treatment and one was hospitalised. None of them attended ambulatory family therapy or another group therapy. Homeopathy and hypnotherapy were also recorded once each.

Seven of the ten participants took medication such as aripiprazole, sertraline, escitalopram and benperidol. Two participants took complementary or alternative medicine: St. John's wort and homeopathy.

Table 1.

Results per participant of the Kafka 2010 criteria for hypersexual disorder.

\begin{tabular}{|l|c|c|c|c|c|c|c|c|}
\hline & A & A1 & A2 & A3 & A4 & A5 & B & C \\
\hline Participant 1 & 1 & 1 & 1 & 1 & 1 & 1 & 1 & 0 \\
\hline Participant 2 & 1 & 1 & 1 & 1 & 1 & 1 & 1 & 0 \\
\hline Participant 3 & 1 & 1 & 1 & 1 & 1 & 1 & 1 & 1 \\
\hline Participant 4 & 1 & 1 & 1 & 1 & 1 & 1 & 1 & 1 \\
\hline Participant 5 & 1 & 0 & 1 & 1 & 1 & 1 & 1 & 1 \\
\hline Participant 6 & 1 & 1 & 1 & 1 & 1 & 1 & 1 & 0 \\
\hline Participant 7 & 1 & 1 & 1 & 1 & 1 & 0 & 1 & 1 \\
\hline Participant 8 & 1 & 1 & 1 & 0 & 1 & 1 & 0 & 0 \\
\hline Participant 9 & 1 & 1 & 1 & 1 & 1 & 1 & 1 & 0 \\
\hline Participant 10 & 1 & 1 & 1 & 1 & 1 & 1 & 1 & 1 \\
\hline
\end{tabular}

(1 = participant meets criterion, 0 = participant does not meet criterion). 


\section{Satisfaction with treatment}

Satisfaction with treatment ranged from neutral to very satisfied. In this study, participants had also the possibility to comment. Most comments were positive: the healthcare workers did not judge or condemn. The participants experienced a lot of understanding, help and support. The 12-step program was evaluated as very supportive. One person claimed to have reached a final stabilisation with the 12-step program. One of the reasons is that the support group is much more accessible in case of an emergency.

Homeopathy and hypnotherapy were perceived as positive. The importance of complementarity was emphasised. Some indicated that psychiatrists behaved too formally, they experienced a lack of involvement and participants found a 20-minute consultation too short.

Some were not satisfied with the treatment in hospitals, others found the results obtained with a sexologist and psychologist insufficient. Shame, taboo and sensationalism were perceived as negative elements concerning the treatment. Some complained about the high cost of several years of treatment.

\section{Discussion}

The low response rate is not surprisingly because the target group was probably cautious and restrained. The discontinuity of anonymity and the fear of stigmatisation is not to be underestimated. The selfishness that accompanies addiction and perhaps also hypersexual disorder, may also play a role. The contact persons indicated that mainly the long-time members participated in the research. In order to achieve a larger study group, more time is needed to build a trusted relationship with the participants by proxy of the contact persons.

\section{Demographics}

All participants were male. From the epidemiological studies we know that the majority is male with a ratio between one in three, to one in five (8).

The average age of the study group was 48 years. In a similar study, the participants all belonged to the age group between 20 and 29 years (14). This difference in average age seems rather a selection bias problem of that specific study than a significant difference with our study. Our participants were recruited in support groups whereas the latter study recruited by advertisements. However, the contact persons indicated that the population attaining the support groups is getting older and that young members are less interested to attend a meeting every week. They prefer quick result.

One participant refused to answer the question on ethnicity. Immigrants are rare in the support groups. For immigrants hypersexual disorder is a lot harder to endure than for natives because of cultural aspects (14). Religion was not questioned in our study. Questions about religion are probably delicate for the participants. Nevertheless these questions are interesting because religion would play an important role in the perception of hypersexual disorder and in the treatment. A religious person would be faster to accept helplessness and therefore attend faster aid (13). One of the steps of the pro- gram is a search for spirituality. In non-religious people this is a taboo but for religious people this is an additional reason to join.

According to one study, about $50 \%$ of people with hypersexual disorder are homosexual or bisexual (13). In our study all participants (except one) were heterosexual. This reflects better the sexual orientation in the general population. It seems to be speculation to suggest that hypersexual disorder is more common among homosexuals or bisexuals. A fortiori, there is almost no epidemiological evidence about the prevalence of hypersexual disorder among groups with a different sexual orientation.

On average, the participants had a degree of non-university higher education. This degree is somewhat higher than in the general population. However, this educational level might be biased by the fact that participants were recruited in support groups. It is known that well-educated patients are more likely to attend support groups. This was at least confirmed for cancer patients (20).

Seven participants had no partner. This is probably related to difficulties in sexual relationships and the pressure on intimacy.

\section{Criteria for hypersexual disorder}

Only five of the 10 participants met the criteria of Kafka. For the others, the diagnosis was possible but not confirmed by the questionnaire. It is not sure that all participants should attend the support groups because the diagnosis of hypersexual disorder was not confirmed for all of them. Before attending a support group the diagnosis of an underlying psychiatric or neurologic disorder should be excluded. Otherwise precious time can be lost in a support group.

The five participants not meeting the Kafka criteria suffered from hypersexual disorder, mainly related to substance addiction. This is in line with the expected comorbidity. Substance addiction would occur in 39 to $45 \%$ of the hypersexual disorder population. For these participants, one cannot exclude that the substance dependence is the primary disorder. The question is whether this criterion is necessary. Dependence of substances and behaviors may have the same aetiology and pathogenesis, making a strict differential diagnosis unnecessary to be able to provide a proper treatment.

The distribution of sexual activity in our study reflects relatively well the results from the literature. In the literature review masturbation (35 to $73 \%$ ) and pornography (49 to 51\%) were the leading activities, followed by sex with consenting adults (13 to 70\%) (1, 5-7).

Masturbation and pornography were reported by all participants in our study. Six participants had contacts with consenting adults. Having cybersex, prostitution and visiting strip clubs were not reported in previous studies. Respectively four and three participants of our study mentioned this. Probably it is useful to add these common sexual activities in further research.

Only one participant reported phone sex. This low proportion corresponds with other studies were for example 1 in 36 participants reported phone sex (14). The other reported behaviors are not included in previous research. 


\section{Diagnosis}

Only in three participants the diagnosis was made by a professional healthcare worker, which is regrettable. For the three cases this was a psychologist - other healthcare professionals were not listed. In all other cases, the diagnoses were made by themselves, their partner or the support group. Previous studies show that patients mainly seek help from psychiatrists, psychologists, lawyers or religious people (15).

From the literature we know that the average age of onset of hypersexual disorder is 18 years (16). In our study, the average onset age is also 17.5 years and ranged between 4 and 24.5 years. It is remarkable that some participants show the first signs of the disorder at a very young age. A similar young age of onset was also described in patients with (other) obsessive-compulsive disorder (21).

But most remarkable is that the diagnosis was on average only made 20 years after the onset of the problem. Many have undergone a long ordeal before they received any help. More attention for the detection and diagnosis of such problems and the possible underlying psychiatric or neurological disorder is desirable in primary healthcare (22).

\section{Treatment}

The strong involvement of the family physicians is striking. Eight family physicians were informed about the diagnosis and in half of the cases they were involved in the treatment.

Sexologists can offer help mainly from the second year of treatment. Four participants of our study were all already treated by a sexologist and often also by a psychologist. Five participants were consulting a psychiatrist and received medication.

All participants received - on top of the support group also another treatment. Nine participants received individual psychotherapy. This combination is the recommended treatment.

None of the participants with a partner followed no relationship therapy. This is optionally recommended from the second year of treatment but the effectiveness is not documented. Two other participants followed relationship therapy with a former partner. An online therapy is not discussed in the literature.

Only two of the ten patients received an SSRI as it is recommended. SSRI are the first choice drugs for hypersexual disorder $(15,17)$. Other patients received atypical psychotics, neuroleptics and homeopathic preparations which are not recommended. However, there is almost no evidence for the treatment of hypersexual disorder.

Before the diagnosis of hypersexual disorder is made, any other underlying psychiatric and neurological disorder should be excluded. Therefore, a consultation with a physician before the start of a treatment in a support group or with a psychologist or a sexologist is recommended.

The participants were positive about their treatment. This finding is probably not representative, since most of the participants attended the support groups for a long time. They are more likely to continue the treatment and therefore are more satisfied. They indicated that the sup- port groups had a considerable added value in their treatment. Participants were generally satisfied about the psychologists, psychiatrists and sexologists. However, some participants were dissatisfied about them.

\section{Weaknesses and future research}

The statistical significance of this research is very limited, due to the small number of participants. However, this research may contribute to the awareness of health care workers for hypersexual disorders. They should receive a training to firstly detect such patients and secondly to refer them for treatment. But primary health care workers also have the very import task to inform and follow-up these patients.

The number of participants was limited for this research. The short inclusion period on the one hand and the inability to directly recruit subjects on the other hand probably played a major role. The number of questions in the survey was deliberately limited to facilitate participation. The anonymity of the participants was absolutely guaranteed, according to their explicit desire. Tendentious questions were avoided to prevent negative reactions and feelings.

Information about the presence of other psychiatric disorders and risky behavior would have been useful. More detailed questions on the third criterion of Kafka were preferable. This would have allowed us to detect hypersexual behavior secondary on substance abuse.

Meanwhile, the American Psychiatric Association (APA) investigated whether or not hypersexual disorder should be included in the DSM-V. In April 2012, the Kafka criteria were adapted again and finally included in chapter III (= appendix) of the new DSM-V. Criteria included in this chapter require more research and evidence.

We cannot estimate how many of our participants would be diagnosed with the adapted criteria because question A was adapted and more detailed information on the third criterion, including manic episodes and general medical conditions are needed.

Further research to illuminate the cause, the diagnosis and the treatment of hypersexual disorder is needed. For future research, a qualitative methodology such as focus group research should be considered.

\section{Conclusions}

It seems very difficult to motivate the members of anonymous support groups to participate in research. In our study, five out of the 10 participants met the criteria of Kafka for hypersexual disorder. For the others the diagnosis was possible but not confirmed by the questionnaire.

The method of diagnosis is far from optimal. This is certainly due to a lack of well-defined criteria for the diagnosis and validated diagnostic instruments. Primary care workers should be sensitised to consider the diagnosis much faster. Today, on average, it takes 20 years before a diagnosis is made. Especially concerning the pharmacological treatment, there seems to exist a lot of uncertainty. At the moment, a combination of medication, support groups and individual psychotherapy is the recommended treatment. 
The questionnaire consists mainly of multiple choice and yes/no questions. You can always indicate an answer.

If multiple answers are possible this is indicated. If none of the answers apply to you, please indicate the most appropriate answer.

May I ask you to fill in the questionnaire completely.

1a. Gender:
(a) Man
․ Woman

1b. Age: in years

1c. Ethnicity:
C Caucasian
口 Negroid
a Asian
- Hispano American
ㅁ Other:

1d. Highest qualification attained:
- Primary education or no diploma
a Secondary education
- Higher non-university education
University

1e. Sexual orientation:
口 Heterosexual
- Homosexual or lesbian
口 Bisexual

1f. Relationship status:
口 In a relationship

a Single

2.A0. I had for years months lots of sex, sexual fantasies and/or a great need for sex (approximate)

2.A1. The time I spent on sex, sexual fantasies and/or a great need for sex repeatedly brought other important (non sexual) goals, activities and commitments into question YES/NO

2.A2. I repeatedly had lots of sex, sexual fantasies and/or a great need for sex in response to sadness, anxiety, depression, boredom or irritability YES/NO

2.A3. I repeatedly had lots of sex, sexual fantasies and/or a great need for sex in response to stressful events YES/NO

2.A4. I have repeatedly tried to control or reduce the amount of sex, sexual fantasies and/or a great need for sex, but failed YES/NO

2.A5. I have repeatedly had sex while there was a risk that this would lead to physical or emotional harm for myself or others YES/NO

2.B. My social life, hobbies or other important areas suffered from the frequency and intensity of sex, sexual fantasies and/or the need for sex YES/NO

2.C. I was addicted to substances such as drugs, alcohol or medication in the period in which I had much sex, sexual fantasies and/or a great need for sex YES/NO

2.S. My sexual activity took place primarily on the following areas: (multiple answers possible)

\author{
प Masturbation \\ a Pornography \\ 口 Sex with consenting adults \\ a Cybersex \\ a Phone sex \\ Strip clubs \\ O Other:
}

3a. My diagnosis was first made by:

- Family physician

a Psychologist

- Sexologist

口 Psychiatrist

Other:

- In addition to the support group I have consulted no other care providers

3b. The diagnosis was made years months ago (approximately).

4c. I have a family physician YES/NO

4c1. If so, my family physician is aware of my diagnosis YES/NO

4d. I am/was treated by the following healthcare professional(s): (multiple answers possible)

ㄱamily physician

- Psychologist

(a) Sexologist

a Psychiatrist

Other:

I In addition to the support group, I was treated by no one else

4e. My treatment consists/consisted, in addition to the support group, of:

- Ambulatory individual psychotherapy

a Ambulatory relational therapy

a Ambulatory family therapy

Ambulatory group therapy other than the support group

- Hospitalisation in a (psychiatric) hospital

- Hospitalisation in a specialised institute

口 Online treatment

Other:

4f. My treatment includes medication YES/NO

4f1. If so, which?

4g. Are you satisfied with the treatment you receive?

a Not at all satisfied

R Rather not satisfied

- Not satisfied, not dissatisfied

Rather satisfied

a Completely satisfied

4g1. Why? 


\section{ACKNOWLEDGEMENT}

The authors are grateful to David Proot for the English editing and the participants of the support groups for the great cooperation and the fascinating conversations about their world.

\section{REFERENCES}

1. Kafka M. Hypersexual disorder: a proposed diagnosis for DSMV. Arch Sex Behav. 2010; 39:377-400.

2. American Psychiatric Association. Diagnostic and Statistical Manual of Mental Disorders, Fourth Edition. 1994: 538-621.

3. Schneider J, Irons R. Assessment and treatment of addictive sexual disorders: relevance for chemical dependency relapse. Subst Use Misuse. 2001; 36:1795-1820.

4. http://www.dsm5.org/proposedrevision/pages/proposedrevision. aspx?rid=415\# 2012, march 2. DSM-V Development. American Psychiatric Association.

5. Mick T, Hollander E. Impulsive-compulsive sexual behavior. CNS Spectr. 2006; 11:944-955.

6. Reid R, Carpenter B. Exploring relationships of psychopathology in hypersexual patients using the MMPI-2. J Sex Marital Ther. 2009; 35:294-310.

7. Dodge B, Reece M, Cole S, Sandfort T. Sexual compulsivity among heterosexual college students. J Sex Res. 2004; 41:343-350.

8. Coleman E. Is your patient suffering from compulsive sexual behavior? Psychiatr Ann. 1992; 22:320-325.

9. Langström N, Hanson K. High rates of sexual behavior in the general population: correlates and predictors. Arch Sex Behav 2006; 35:37-52.

10. Carnes P, Schneider J. Recognition and management of addictive sexual disorders: guide for the primary care clinician. Lippincotts Prim Care Pract. 2000; 4:302-318.

11. Kelly B, Bimbi D, Nanin J, Izienicki H, Parsons J. Sexual compulsivity and sexual behaviors among gay and bisexual men and lesbian and bisexual women. J Sex Res. 2009; 46:301-308.
12. Winters J, Christoff K, Gorzalka B. Dysregulated sexuality and high sexual desire: distinct constructs? Arch Sex Behav. 2010; 39:1029-1043.

13. Skegg K, Nada-Raja S, Dickson N, Paul C. Percieved "out of control" sexual behavior in a cohort of young adults from the Dunedin Multidisciplinary Health and Development Study. Arch Sex Behav. 2010; 39:968-978.

14. Black D, Kehrberg L, Flumerfelt D, Schlosser S. Characteristics of 36 subjects reporting compulsive sexual behavior. Am J Psychiatry 1997; 154:243-249.

15. Guay D. Drug treatment of paraphilic and nonparaphilic sexual disorders. Clin Ther. 2009; 31:1-31.

16. Bird M. Sexual addiction and marriage and family therapy: facilitating individual and relationship healing through couple therapy. J Marital Fam Ther. 2006; 32:297-311.

17. Bradford J. The neurobiology, neuropharmacology, and pharmacological treatment of the paraphilias and compulsive sexual behavior. Can J Psychiatry 2001; 46:26-34.

18. Reid R, Garos S, Carpenter B. Reliability, validity and psychometric development of the Hypersexual Behavior Inventory in an outpatient sample of men. Sexual Addiction \& Compulsivity. 2011; 18:30-51.

19. Miner M, Coleman E, Center B, Ross M, Rosser B. The compulsive sexual behavior inventory: psychometric properties. Arch Sex Behav. 2007; 36:579-587.

20. Bauman L, Gervey R, Siegel K. Factors associated with cancer patients' participation in support groups. J Psychosoc Oncol. 1992; 10:1-20

21. Lochner C, Hemmings SM, Kinnear CJ, et al. Cluster analysis of obsessive-compulsive spectrum disorders in patients with obsessivecompulsive disorder: clinical and genetic correlates. Compr Psychiatry. 2005; 46:14-19.

22. Kaplan MS, Krueger RB. Diagnosis, assessment, and treatment of hypersexuality. J Sex Res. 2010; 47:181-198. 